\title{
Análisis de la codeleción 1p19q como marcador pronóstico en pacientes con glioblastoma primario
}

\section{Analysis of $1 p 19 q$ codeletion as a prognostic marker for patients with primary glioblastoma}

\author{
- Andrés Felipe Cardona1'-3, León Darío Ortiz $z^{3,4}$, Henry Becerra', Enrique Jiménez-Hakim5, Fernando Hakim5, \\ Juan Armando Mejía ${ }^{5}$, Sonia Bermúdez ${ }^{6}$, Nicolás Useche ${ }^{6}$, Carlos Emilio Restrepo ${ }^{5}$, Silvia Serrano ${ }^{2}$, Hernán Carranza1,2,

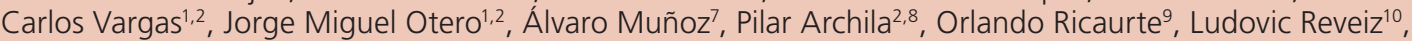 \\ July Katherine Rodríguez², Andrés Acevedo"11, Germán Peña ${ }^{5}$, Diana Torres ${ }^{2,12}$ \\ 'Grupo Oncología Clínica y Traslacional, Instituto de Oncología, Fundación Santa Fe de Bogotá (Bogotá, Colombia) \\ Fundación para la Investigación Clínica y Molecular Aplicada del Cáncer (FICMAC); investigador asociado ONCOLGroup. \\ Investigador asociado Red Latinoamericana de Neurooncología (RedLANO). \\ "Departamento de Oncología Clínica, Sección Neurooncología, Instituto de Cancerología, Clínica Las Américas (Medellín, Colombia). \\ Departamento de Neurocirugía, Fundación Santa Fe de Bogotá (Bogotá, Colombia). \\ Departamento de Imágenes Diagnósticas, Sección Neurorradiologia, Fundación Santa Fe de Bogotá (Bogotá, Colombia). \\ Grupo Radioterapia, Instituto de Oncología, Fundación Santa Fe de Bogotá (Bogotá, Colombia). \\ Departamento de Patología, Hospital de San José (Bogotá, Colombia). \\ Departamento de Patología, Universidad Nacional de Colombia (Bogotá, Colombia). \\ "Grupo Hematología y Trasplante de Médula Ósea, Instituto de Oncología, Fundación Santa Fe de Bogotá (Bogotá, Colombia). \\ ${ }^{12}$ Instituto de Genética Humana, Pontificia Universidad Javeriana (Bogotá, Colombia).
}

\section{Resumen}

Introducción: La deleción de los cromosomas 1p y/o 19q se ha relacionado con el pronóstico y la quimiosensibilidad en pacientes con oligodendrogliomas anaplásicos y glioblastomas (GB) secundarios. No obstante, el pronóstico de estas alteraciones en Colombia es desconocida.

Materiales y métodos: Presentar un análisis detallado de la supervivencia libre de progresión (SLP) y global (SG) de 38 pacientes con GB primario en quienes se realizó un estudio exploratorio sobre el impacto de las traslocaciones $1 p / 19 q$ evaluadas por reacción en cadena de polimerasa (PCR).

Resultados: La media para la edad fue de 60 años ( $D E \pm 1$ ) y la distribución por sexo fue homogénea (19 hombres y 19 mujeres). La mediana de SG según el estado del $1 p$ y del $19 q$ fue: $1 p(+)(n=10) 23.6$ meses (rango 10.8-36.4)/1p(-) $(n=28)$ 15.8 meses $(2.6-9.0)(p=0.04)$ y $19 q(+)(n=12) 7.6$ meses $(4.4-10.7) / 19 q(-)(n=26) 10.43$ meses $(1.2-19.6)(p=0.82)$. De igual forma, entre quienes tuvieron el $1 p(+)$, la mediana de SLP fue de 9.73 meses (2.6-53) versus el $1 p(-) 3.97$ meses (4.315.1) $(p=0.053)$.

Conclusión: En una serie limitada de pacientes con GB primario, se encontró que el $1 p(+)$ influyó sobre la SG y la SLP. Estos hallazgos requieren una validación prospectiva, pero sugieren que la deleción del $1 p$ puede afectar el pronóstico de los gliomas de alto grado tratados con quimiorradiación.

Palabras clave: 1p/19q, glioblastoma, temozolamida, glioma de alto grado, respuesta, supervivencia.

\begin{abstract}
Background: Deletions on chromosomes $1 p$ and $19 q$ have been shown to correlate with prognosis and chemosensitivity in anaplastic oligodendrogliomas and secondary glioblastoma (GB). However, the impact of these alterations over the main outcomes in Colombian patients is unclear.

Materials and methods: To present a detailed analysis of a cohort of 38 patients with primary GB who participated in an exploratory analysis of $1 \mathrm{p} 19 \mathrm{q}$ translocations documented by polimerase chain reaction (PCR). Data include the analysis of progression free survival (PFS) and overall survival (OS).

Results: Mean age was $60(S D \pm 1)$ and distribution by gender was homogenous (19 males and 19 females). OS according to the state of $1 p$ and $19 q$ was: $1 p(+)(n=10) 23.6$ months (range 10.8-36.4)/1p(-) $(n=28) 15.8$ months $(2.6-9.0)(p=0.04)$ and $19 q(+)(n=12) 7.6$ months $(4.4-10.7) / 19 q(-)(n=26) 10.43$ months $(1.2-19.6)(p=0.82)$. Likewise, for those having $1 \mathrm{p}(+)$, PFS was 9.73 months (2.6-53) compared to $1 \mathrm{p}(-)$ which was 3.97 months $(4.3-15.1)(p=0.053)$.

Conclusion: We found that $1 \mathrm{p}(+)$ influenced OS and PFS in a limited series of patients with primary GB treated with chemo/ radiotherapy in Colombia. Such findings must be validated prospectively but the results suggest that $1 \mathrm{p}$ affected the prognosis of non-selected with GB.
\end{abstract}

Key words: 1p/19q, glioblastoma, temozolamide, high grade glioma, response, survival 


\section{Introducción}

La clasificación histológica de la Organización Mundial de la Salud (OMS) constituye el estándar actual para determinar el pronóstico y seleccionar las intervenciones terapéuticas para los tumores cerebrales de estirpe glial'. El glioblastoma (GB) es el tumor primario más frecuente y agresivo entre los adultos, representando entre el $12 \%$ y $15 \%$ de todas las neoplasias intracraneales. A pesar del tratamiento multimodal disponible, que incluye la cirugía radioterapia y quimioterapia, el pronóstico de esta entidad nosológica continúa siendo pobre, con una mediana de supervivencia global (SG) que oscila entre los 12 y 14 meses y una mortalidad estimada a los dos años del $80 \%{ }^{1}$.

En algunos casos, se pueden lograr supervivencias más prolongadas, especialmente en la población de sujetos menores de 60 años'. También se han identificado algunos factores clínicos relacionados con la evolución de la enfermedad, entre los que cabe mencionar la edad temprana de presentación, el estado funcional al diagnóstico, el uso de quimioterapia adyuvante, la extensión de la resección y el estado de metilación de la metil-guanina-metil-transferasa (MGMT). Sin embargo, estas variables son insuficientes para definir el pronóstico de una neoplasia que presenta heterogeneidad genotípica².

Con la aparición de nuevos marcadores moleculares, se ha podido caracterizar, al menos en parte, el comportamiento de los tumores cerebrales. El conocimiento progresivo de la génesis tumoral ha permitido comprender las vías implicadas en el desarrollo, progresión y cambio en la gradación de los tumores gliales de alto grado². En los GB, se han encontrado alteraciones asociadas con la amplificación y sobreexpresión de los genes EGFR (epidermal growth factor receptor), CDK4 (cyclin-dependent kinase 4) y MDM2 (murine doble minute 2), además de la inactivación de varios genes supresores de tumor localizados en los cromosomas 9 (CDKN2A) P16), 10p, 10q (incluyendo el gen PTEN/MMAC1), $13 q, 17 p(p 53), 19 q$ y $22 q^{3,4}$.

Uno de los descubrimientos más importantes en la genética de los tumores cerebrales ha sido la diferenciación entre los oligodendrogliomas anaplásicos y los GB; en los primeros, se han documentado pérdidas alélicas en los cromosomas $1 p$ y $19 q$ (60\% al $80 \%$ de los casos), como resultado de una translocación no balanceada $t(1 ; 19)(q 10 ; p 10)^{5-8}$, que se correlaciona con una mayor sensibilidad a los agentes alquilantes y a la radioterapia, eventos que permiten predecir la SG 2,9,10.

Múltiples estudios han analizado el compromiso de grandes regiones de microsatélites en GB sin focalizarse en genes específicos. La complejidad de la estructura centromérica de la región cromosómica $1 \mathrm{p}$ ha sido un obstáculo para el mapeo de los genes supresores tumorales localizados en esta área. Boulay y colaboradores describieron deleciones en el cromosoma 1p en una población de 118 pacientes con GB secundario, demostrando que las pérdidas parciales o totales en el brazo corto del cromosoma 1 permiten subdividir la enfermedad en tres categorías que confieren diferentes perfiles pronósticos; estas son: las teloméricas (47\%), las intersticiales $(29 \%)$ y las centroméricas (24\%).

Los mismos autores diseñaron 10 haplotipos diferentes para correlacionarlos con la SG de los pacientes, encontrando que la pérdida del marcador centromérico D1S2696, localizado en el intrón 12 del gen NOTCH2 (neurogenic locus notch homolog protein 2), se asoció de forma significativa con un curso favorable de la enfermedad ( $p=0.0175)$, en tanto que la pérdida del $19 q$ concomitante con el compromiso de la heterocigosidad del $1 p$ no influyó sobre este desenlace. También se desarrolló una combinación para determinar duplicaciones pericéntricas en 1q21 y NOTCH2, hallando algún grado de relación con la supervivencia a dos años ${ }^{8}$.

Este estudio presenta un análisis del pronóstico y desenlaces encontrados en 38 pacientes colombianos con el diagnóstico de GB primario según el perfil de la codeleción 1p19q.

\section{Materiales y métodos}

El análisis incluyó una cohorte de pacientes con GB tratados con biopsia cerebral guiada bajo neuronavegación o con resección óptima ( $\geq 95 \%$ ) o subóptima de las zonas comprometidas, descartando aquellas con necrosis o sangrado. Todos los casos fueron seleccionados durante un período de dos años y se obtuvo consentimiento informado para la realización de los estudios y su inclusión en el registro previa aprobación del estudio por un comité de ética independiente. 


\section{Extracción de ADN}

La extracción del ADN se hizo a partir de dos secciones de 10 micras de tejido tumoral embebido en parafina, obtenido mediante microdisección manual y utilizando un kit de extracción dispuesto para tal fin (Macherey-Nagel, Düren, Alemania). Después de la remoción de la parafina y de la exposición nocturna del ADN a proteinasa $K$, se obtuvo el material genético, siguiendo pasos de incubación y centrifugación. Se determinó la concentración de ADN mediante espectrofotometría, para asegurar que hubiese suficiente cantidad del material (logrando una concentración fija de alrededor de $30 \mathrm{mcg} / \mathrm{ml}$ ). Todas las muestras se almacenaron a $-20^{\circ} \mathrm{C}$ hasta el momento de su análisis.

\section{Reacción en cadena de polimerasa (PCR)}

Para la realización del análisis de la pérdida de heterocigosidad, se amplificaron ocho secuencias de microsatélites mediante PCR (tabla 1). Cuatro de estos se localizaron en el cromosoma $1 p$ y cuatro en el 19q. Para amplificar los ocho microsatélites, se estandarizaron tres reacciones de PCR (2 PCR dúplex y 1 PCR multiplex) que se llevaron a cabo en un volumen final de $15 \mu \mathrm{l}$, utilizando una mezcla maestra específica para PCR multiplex (Qiagen, Mannheim, Alemania), incluyendo 100 ng de ADN por reacción.

El termociclador se programó siguiendo un patrón estándar para la denaturación inicial a $95^{\circ} \mathrm{C}$; el anillaje de los cebadores se hizo a una temperatura específica durante un minuto con extensión a $72^{\circ} \mathrm{C}$ por el mismo tiempo. Dentro de las PCR MP4.1 y MP4.2 (ambas con

Tabla 1. Secuencia de los primers y longitud de los fragmentos obtenidos

\begin{tabular}{|c|c|c|c|}
\hline Primer $5^{\prime}$ & Modif. ${ }^{1}$ & Secuencia & Longitud $^{2}$ \\
\hline D1S508-U & FAM & AgCTggggAATATATgTNTCATAT & \\
\hline D1S508-L & No & TTggCCCgTTgTTggTTgAA & $115 \mathrm{pb}^{3}$ \\
\hline D1S2734-U & FAM & CCTTgTCACATggCCTCATA & \\
\hline D1S2734-L & No & CTggCACTCAgACCTCAAAA & $210 \mathrm{pb}$ \\
\hline D1S214-U & FAM & gCTgAggCAggAgAATAgTT & \\
\hline D1S214-L & No & ACCTAgAAgTgggATTgCTg & $200 \mathrm{pb}$ \\
\hline D1S199-U & FAM & AgCCATgATCATgACACTACA & \\
\hline D1S199-L & No & gACCATgTgCTCCgTAAATA & $130 \mathrm{pb}$ \\
\hline D19S219-U & HEX & AggTTgCAgTgAgCCAAgAT & \\
\hline D19S219-L & No & TgTTाTgTggCTgTgCTCCT & $160 \mathrm{pb}$ \\
\hline D19S412-U & HEX & gAgCgACAgAATgAgACTCT & \\
\hline D19S412-L & No & ACCTCATgTgTCTCCTCCTT & $130 \mathrm{pb}$ \\
\hline D19S596-U & HEX & AATCCgAgAggTgggAgTTT & \\
\hline D19S596-L & No & TTTTTggAgCCAgAgCCACT & $215 \mathrm{pb}$ \\
\hline D19S112-U & HEX & gCCAgCCATTCAgTCATTTgAAg & \\
\hline D19S112-L & No & gCCAgCCATTCAgTCATTTgAAg & $120 \mathrm{pb}$ \\
\hline \multicolumn{4}{|c|}{$\begin{array}{l}\text { 'Modif:: modificación. } \\
{ }^{2} \text { Longitud: la longitud aproximada de cada fragmento puede variar entre individuos. } \\
{ }^{3} \text { pb: pares de bases. }\end{array}$} \\
\hline
\end{tabular}

una temperatura de anillaje de $61^{\circ} \mathrm{C}$ ), se amplificaron los microsatélites D1S214 y D19S219, y D1S199 y D19S112, respectivamente. Mientras la reacción de PCR MP8 (temperatura de anillaje de $55^{\circ} \mathrm{C}$ ) amplificó los microsatélites D1S2734, D1S508, D19S412 y D19S596.

\section{Análisis por GeneScan}

Para el análisis de la pérdida de heterocigosidad, los productos de la PCR fueron separados en el equipo AbiPrism (Applied Biosystems, UK) usando $2 \mu \mathrm{l}$ por cada reacción de PCR que se mezcló con $1 \mu$ de GeneScan-500 ROX de tamaño estándar (Applied Biosystems, Cheshire, Reino Unido) y con $10 \mu \mathrm{l}$ de formamida desionizada. Después de la desnaturalización, se añadieron $10 \mu \mathrm{l}$ de la mezcla en una bandeja de 324 pozos y se hizo la lectura de los resultados con el programa GeneScan, que permite la detección fluorimétrica de múltiples locis.

\section{Análisis estadístico}

La información extraída de las historias clínicas fue valorada por dos investigadores, que registraron los datos en un formato diseñado para el estudio. Posteriormente, estos se digitaron en una base de datos y se analizaron usando el paquete estadístico SPSS v19.0 (SPSS Inc. Chicago, IL, EE. UU.).

Se analizaron diversas variables demográficas, la SG, la SLP, y otras que se consideraron tenían implicaciones pronósticas. Los resultados se obtuvieron y describieron por medio de la determinación de frecuencias absolutas, relativas, medidas de tendencia central y de dispersión. El cruce de variables se realizó con tablas de contingencia sometidas a pruebas de dependencia y asociación, empleando el estadístico Jl al cuadrado $\left(X^{2}\right)$ o el test exacto de Fisher cuando fue necesario. Para todos los casos, el nivel de significancia fue $p<0.05$.

Las estimaciones de supervivencia se hicieron utilizando el modelo no paramétrico del límite del producto (método de Kaplan-Meier), y las funciones de supervivencia se compararon mediante la prueba log-rank. Con el fin de controlar la confusión, se realizó un análisis multivariado de supervivencia mediante la regresión de Cox, haciendo uso de la prueba de Wald para corroborar la significancia de los parámetros en el modelo. Se verificó gráficamente el supuesto de riesgos proporcionales y los demás se evaluaron mediante el análisis de residuales. 


\section{Resultados}

\section{Características de la población}

Un total de 38 pacientes con edades entre 24 y 75 años (media 50, SD \pm 1 ) fue incluido en el estudio. Diecinueve sujetos fueron mujeres, el $84.2 \%$ eran mayores de 50 años, el $65.8 \%$ de los pacientes tenía un estado funcional basal cuantificado por el índice de Karnofsky mayor del 70\% y el Barthel excedió el 70\% en 23 sujetos. La mediana para el tamaño de la lesión inicial fue $21.7 \mathrm{~mm}$ (rango 14-64 mm), sin diferencias según el sexo $(p=0.27)$, la presencia o no de multicentricidad $(p=0.092)$, la localización de la neoplasia $(p=0.82)$ y el hallazgo de sangrado $(p=0.54)$. No obstante, a mayor tamaño tumoral ( $\geq 30 \mathrm{~mm}$ ), superior el riesgo de compromiso funcional $(p=0.036)$, la necesidad de soporte esteroide $(p=0.028)$ y la extensión al cuerpo calloso $(p=0.001)$.

La tabla 2 presenta las principales características demográficas de la población en estudio. De la totalidad de los sujetos incluidos, el $52.6 \%(n=20)$ no tuvo alteraciones completas o parciales en 1p o 19q. Por el contrario, 7 sujetos tuvieron alguna deleción en el 19q y 2 en ambos. La distribución de estas alteraciones no presentó ninguna asociación con el tamaño tumoral estratificado, el sexo, la presencia de multicentricidad, la afectación del cuerpo calloso y el estado funcional. En contraposición, se documentó una tendencia positiva para la mejor respuesta al tratamiento concomitante

Tabla 2. Características generales de los pacientes con diagnóstico de GB

\begin{tabular}{|l|c|c|}
\hline \multicolumn{1}{|c|}{ Caracteristica } & N & $\%$ \\
\hline Sexo & 19 & 50 \\
Hombre & 19 & 50 \\
Mujer & & \\
\hline Edad & 50 & \\
Media & 1 & \\
SD $( \pm)$ & & \\
\hline Edad por estratos & 32 & 84.2 \\
$>50$ años & 6 & 15.8 \\
\hline 50 años & 25 & \\
\hline Escala funcional de Karnofsky (\%) & 8 & 65.8 \\
$\geq 70$ & 7 & 21.1 \\
$<70$ & 31 & 18.4 \\
\hline Multicentricidad & & 81.6 \\
Sí & 7 & 18.4 \\
No & 30 & 78.9 \\
\hline Infiltración del cuerpo calloso & & \\
Sí & 27 & 71.1 \\
No & 6 & 15.8 \\
\hline Enfermedad residual & 5 & 13.2 \\
Sí & 9 & \\
No & 10 & 23.0 \\
ND & 19 & 50 \\
\hline Compromiso neurológico basal & & \\
Sí & & \\
No & & \\
ND & & \\
\hline
\end{tabular}

con radio y quimioterapia con temozolomida, seguida de temozolomida adyuvante $(p=0.052)$.

\section{Supervivencia global y pronóstico}

El análisis de la pérdida de heterocigosidad para el $1 p$ encontró en la población positiva una SG de 23.6 meses (rango 10.8-36.4), valor que para la negativa fue de 15.8 meses (rango 2.6-9.0) ( $p=0.046)$ (figura 1). Así mismo, la SLP de los pacientes que fueron positivos para el $1 p$ difirió de su contraparte negativa, siendo de

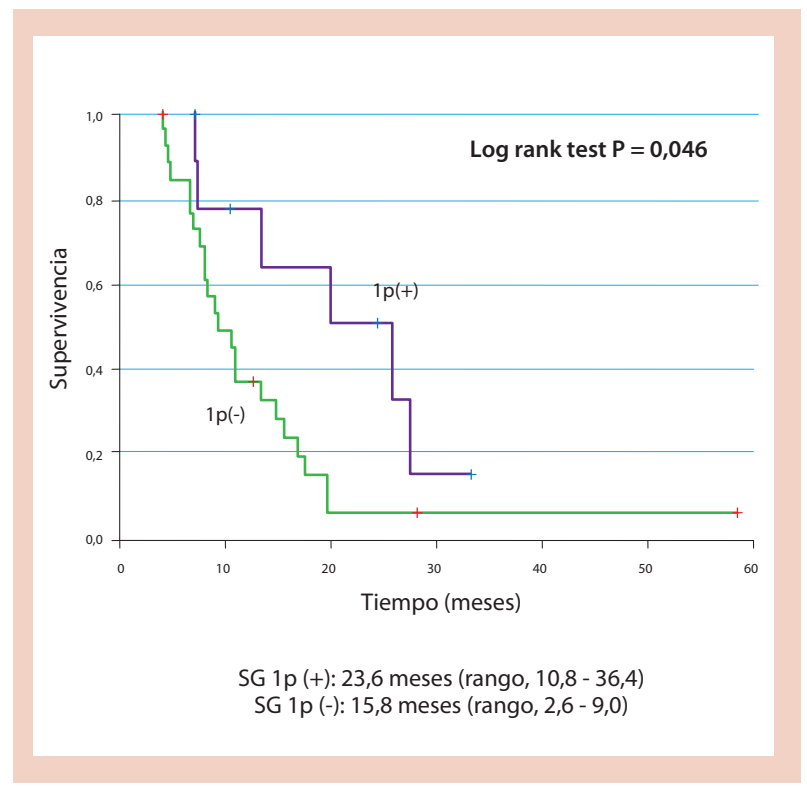

Figura 1. Relación entre la codeleción $1 p$ y la SG de los pacientes en estudio.

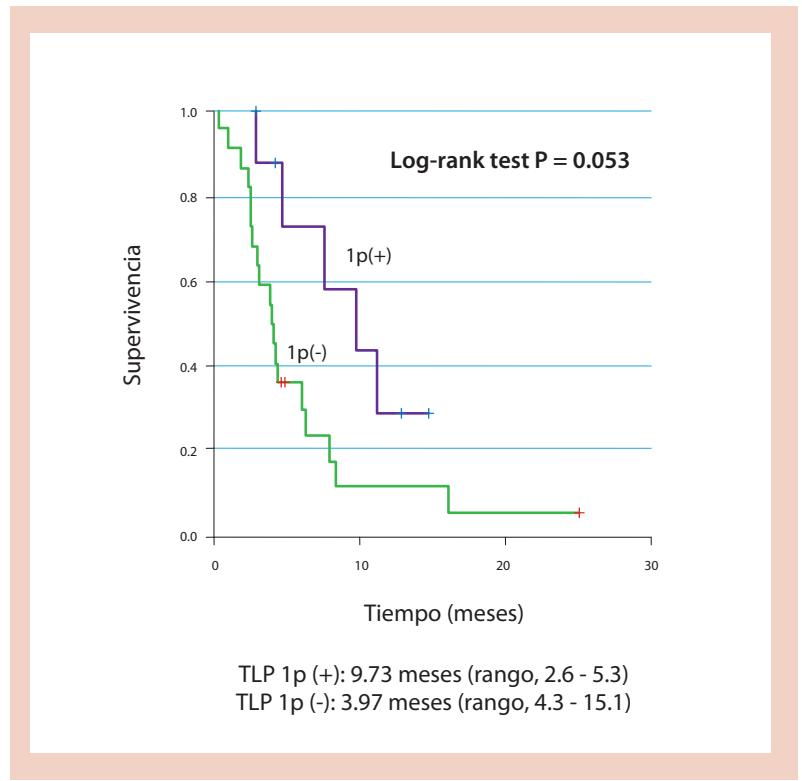

Figura 2. Diferencias para el tiempo libre de progresión entre los grupos con y sin la pérdida de heterocigosidad para $1 \mathrm{p}$. 


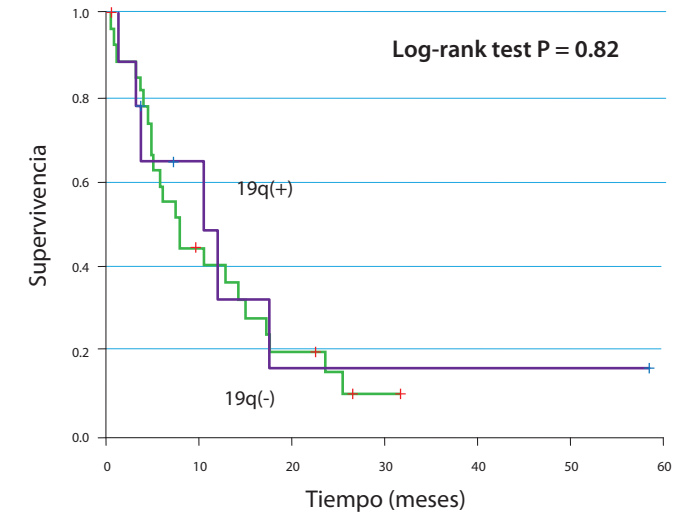

SG 19q (+): 7.6 meses (rango, $4.4-10.7)$ SG $19 q(-): 10.43$ meses (rango, $1.2-19.6)$

Figura 3. Diferencia para la SG respecto de la positividad o negatividad para la pérdida de heterocigosidad del 19q.

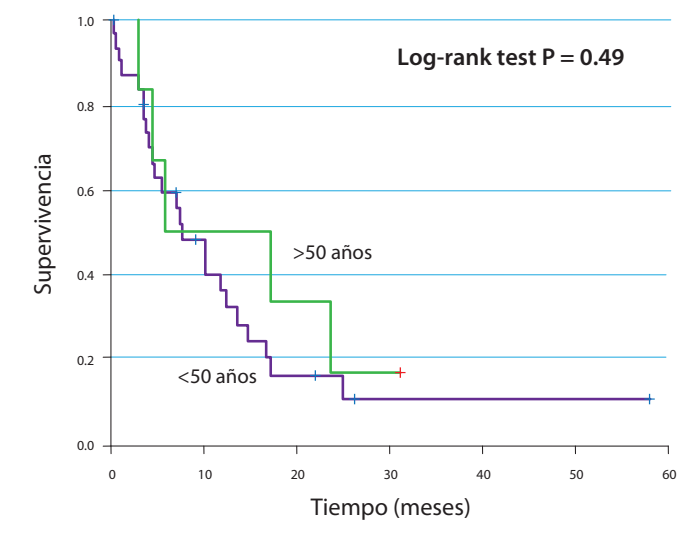

SG >50 años: 5.8 meses (rango, $0.1-21.0$ ) SG $<50$ años: 7.7 meses (rango, $2.8-12.5$ )

Figura 5. Diferencias en la SG según la distribución por la edad.

9.7 meses (rango 2.6-5.3) para el primer grupo y de 3.9 meses (rango 4.3-15.1) para el segundo $(p=0.005)$ (figura 2). El mismo análisis para la deleción 19q mostró entre los pacientes positivos y negativos para la pérdida de heterocigosidad una SG de 7.6 meses (rango 4.410.7) y de 10.4 meses (rango 1.2-19.6), respectivamente $(p=0.82)$ (figura 3).

Al discriminar la SG según el estado positivo para el 1p y 19q, y negativo para ambos, se encontró que la SG para los sujetos que tenían la pérdida del 19q fue de 10.4 meses (rango 1.2-19.6), mientras que para los

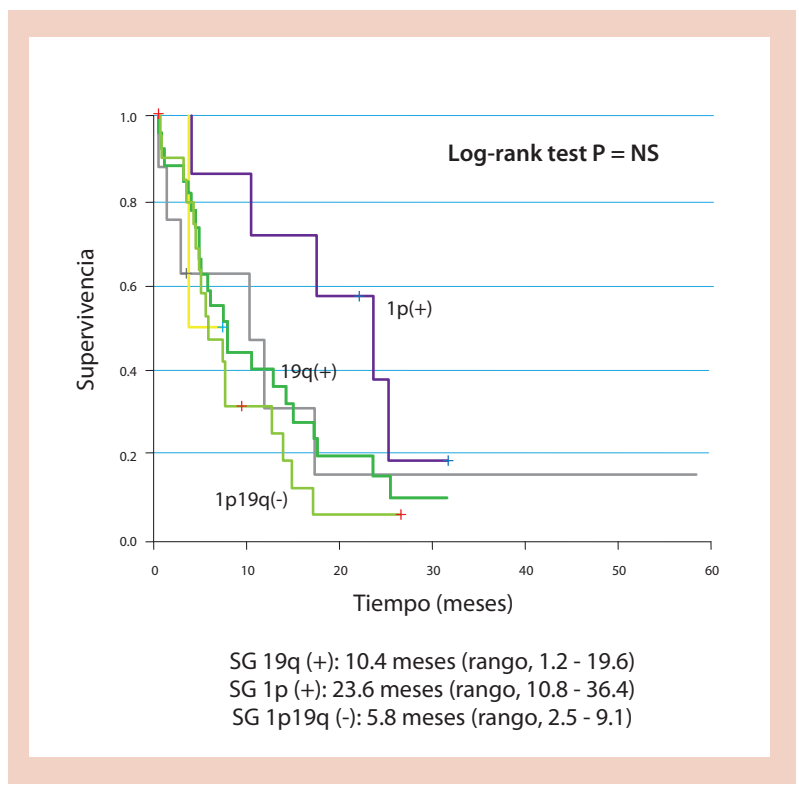

Figura 4. Supervivencia global discriminada por la pérdida de 1p19q y la negatividad para ambos marcadores.

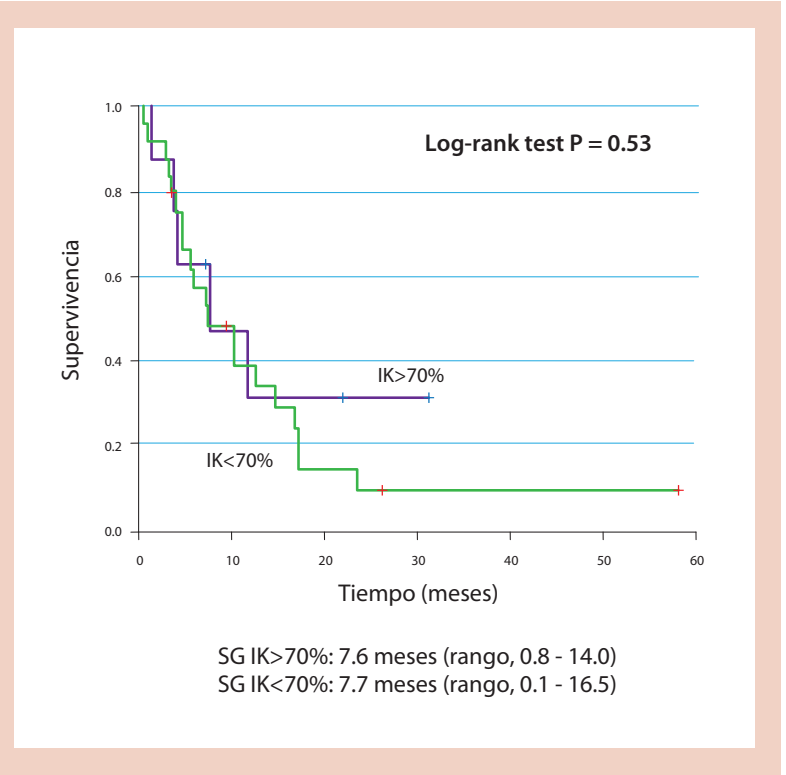

Figura 6. Supervivencia según el estado funcional basal.

positivos en el 1p fue de 23.6 meses (rango 10.8-36.4) $(p=0.02)$. En contraposición, la SG para los pacientes que fueron negativos para el $1 p$ y $19 q$ fue de 5.8 meses (rango 2.5-9.1) (figura 4). Tampoco se halló una diferencia positiva cuando los pacientes se estratificaron según su edad (mayores y menores de 50 años, 5.8 meses vs. 7.7 meses; $p=0.49$ ) (figura 5). El estado funcional basal no influyó sobre la SG, que resultó ser de 7.6 meses (rango 0.8-14.0) para aquellos con un índice mayor del $70 \%$ y de 7.7 meses (rango 0.1-16.5) para los sujetos más comprometidos ( $p=0.53$ ) (figura 6 ). El modelo 
Tabla 3. Modelo de Cox para la supervivencia global (significancia estadística para el análisis de $1 \mathrm{p})$

\begin{tabular}{|l|c|c|c|c|c|c|c|}
\hline \multirow{2}{*}{ Variable } & \multirow{2}{*}{ B } & SE & Wald & Sig. & RR & \multicolumn{2}{c|}{ IC95\% } \\
\cline { 7 - 10 } & & & & & & Inf. & Sup. \\
\hline PCR19q & 99 & 0.47 & 0.55 & 0.45 & 1.4 & 0.56 & 3.58 \\
\hline PCR1p & 0.35 & 0.48 & 4.2 & 0.04 & 2.7 & 1.04 & 6.98 \\
\hline Edad (>50 años) & 0.48 & 0.56 & 0.74 & 0.38 & 1.62 & 0.53 & 4.91 \\
\hline Enfermedad residual & -0.50 & 0.56 & 0.80 & 0.36 & 0.60 & 0.20 & 1.81 \\
\hline Estado funcional & -0.30 & 0.50 & 0.37 & 0.53 & 0.73 & 0.27 & 1.96 \\
\hline
\end{tabular}

de Cox demostró que la única variable que modificó positivamente la SG fue la deleción parcial o completa del $1 p(p=0.004)$ (tabla 3$)$.

\section{Discusión}

Los tumores de estirpe glial de origen astrocitario u oligodendroglial, y aquellos con un patrón mixto son los más frecuentes en los adultos. Suelen tener fenotipos diferentes y cursos clínicos disímiles; desde la introducción de la inmunohistoquímica y el uso de la biología molecular tumoral, su diferenciación es más sencilla y permite seleccionar los tratamientos oncológi$\cos ^{11}$. La codeleción 1p19q se encuentra en la mayoría de los oligodendrogliomas, permitiendo subclasificar este tumor ${ }^{11}$; resultados publicados por el Radiation Therapy Oncology Group (RTOG) y la European Organization for Research and Treatment of Cancer (EORTC) determinaron en pacientes con oligodendrogliomas y oligoastrocitomas anaplásicos que la pérdida parcial de los cromosomas 1 y 19 tiene un impacto significativo sobre la SG, hasta el punto de permitir caracterizar la enfermedad por su genotipo².

Hay información que sustenta que el número de copias de locus específicos podría estar involucrado en mecanismos relacionados con la progresión tumoral y la respuesta al tratamiento. Se ha intentado establecer los genes que se encuentran en zonas particulares del cromosoma 1, evento que ha permitido documentar alteraciones en el $\mathrm{NOTCH} 2$, gen que regula varias vías relacionadas con el neurodesarrollo 8,12 . Aproximadamente el $15 \%$ de los GB presentan algún componente oligodendroglial y una frecuencia variable de las deleciones en $1 p$ y $19 q$, hecho que se correlaciona con una mejor expectativa de vida ${ }^{13,14}$. En general, los GB primarios muestran pérdidas de algunos alelos del brazo corto del cromosoma 1 y del largo del 19 en cerca del $10 \%$ de los casos, en tanto que las pérdidas cromosómicas en el $19 q$ ocurren del $13 \%$ a $32 \%$ y se asocian con una mayor supervivencia ${ }^{14}$.

Un estudio realizado en Turquía que incluyó 40 pacientes con GB encontró que las lesiones primarias presentan alguna aberración genotípica en el $100 \%$ de los casos (1 a 6 por caso), siendo las más frecuentes las deleciones. De forma global, la alteración dominante ocurrió en el cromosoma 10, en donde se documentaron pérdidas parciales en 9 casos $^{12}$. Por otra parte, los cambios alélicos en el 1p y en el 19q solo se hallaron en 10 y 4 casos, respectivamente. Otro análisis que incluyó tumores gliales de 45 pacientes con tumores gliales supratentoriales documentó pérdidas por heterocigosidad en los cromosomas 1 y 19 en el $14 \%$ de los casos $^{2}$.

Ishii y colaboradores describieron la presencia de cambios alélicos en $1 p$ en el $14 \%$ de los pacientes examinados y en el $9.5 \%$ para $19 q$, hallazgos discordantes con la presentación de alteraciones en el 10q (33,3\%). En esta población, los datos se asociaron con un estado positivo para la metilación del promotor de la MGMT (06-metilguanina-DNA-metiltransferasa) $)^{15}$. El análisis de un registro de pacientes con GB y larga supervivencia encontró que cerca del $6 \%$ de los casos presentaba la codeleción 1p/19q, hallazgo que fue similar a lo descrito previamente ${ }^{16}$. El análisis de los casos de Colombia demostró frecuencias superiores para las pérdidas alélicas en ambos cromosomas, reportando la alteración para $1 p$ y $19 q$, en el $26 \%$ y $31 \%$, respectivamente. Estos datos no se asociaron con el estudio de la metilación del promotor de la MGMT realizado en 18 de los casos, de los cuales el 30\% resultó positivo (datos no publicados registro Red Latinoamericana de Neurooncología-RedLANO-).

En población europea, Houilier y colaboradores encontraron una asociación positiva entre la pérdida de heterocigosidad para el $1 p$ y $19 q(p=0.005)$; no obstante, su presencia no modificó el curso de la SG ${ }^{17}$. Por el contrario, Gresner halló una asociación positiva entre las alteraciones de dos de los locus del $1 p$ y la mejoría en la SLP ( $p=0.05)$. En paralelo, el grupo de pacientes con pérdida de la heterocigosidad de este gen tuvo una posibilidad 10 veces mayor de tener un GB secundario ${ }^{18}$.

En América Latina, pocos estudios han discriminado el papel pronóstico de la codeleción 1p/19q en pacientes con GB. Wernersbach y colaboradores estudiaron 88 sujetos de Brasil mediante FISH o por el 
análisis cuantitativo de los microsatélites, encontrando una pérdida aislada del $1 \mathrm{p}$ en 4 casos $(4.5 \%)$ y la del $19 q$ en 5 (5.6\%). Estas frecuencias no favorecieron la mejoría en la supervivencia de la población en estudio, hallazgo disonante con el colombiano, que evidenció en el análisis multivariado una influencia significativa del resultado del $1 p$ sobre la SG ${ }^{14}$. Múltiples estudios han mostrado una relación nula entre el estado del $1 p / 19 q$ respecto de diversas variables clínicas y patológicas. Aunque la frecuencia de las pérdidas en el 1 y $19 q$ es más alta entre los GB secundarios (19\% vs. $7.4 \%$ para el $1 p$ y $20 \%$ vs. $9.4 \%$ para el $19 q$ ), este hallazgo no parece influir sobre los desenlaces primarios $^{18}$.

El presente estudio, el primero diseñado y ejecutado en población colombiana, descubrió que las alteraciones en el 1p pueden repercutir positivamente sobre la SG y SLP, hallazgo biológico que parece constituir un factor pronóstico. No obstante, presenta ciertas limitaciones, dentro de las que cabe mencionar la ausencia de revisión neuropatológica centralizada carente de la búsqueda exhaustiva de fragmentos tumorales que incluyan un patrón glial mixto. De igual forma, no se cuantificó la expresión del OLIG2 (oligodendrocyte transcription factor 2), proteína relacionada con el gen regulador de los progenitores neuroectodérmicos de la región ventral ${ }^{19}$. Su marcación diferencial parece contribuir con la estratificación de los tumores de bajo grado y anaplásicos que tienen un carácter evolutivo dependiente de los oligodendrocitos.

Otros problemas del estudio se sustentan en la incapacidad para evaluar la metilación de genes, como el MGMT implicado en la sensibilidad y resistencia a los tratamientos oncológicos de primera línea. También existen diferentes métodos para el estudio de las alteraciones cromosómicas en el 1p19q, como la evaluación por PCR, el FISH y la HCG (hibridización genómica comparativa), pruebas que demuestran una concordancia que oscila entre el $73 \%$ y $99 \%$ de los casos. En nuestro entorno, es necesario disponer de técnicas costo-efectivas que permitan seleccionar adecuadamente a los pacientes con GB candidatos para recibir quimio y radioterapia. En el futuro, el uso racional y equitativo de estos permitirá proyectar el pronóstico de la enfermedad y predecir la eficacia de las intervenciones².

\section{Referencias}

1. Louis N, Ohgaki H, Wiestler O, et al. World Health Organization classification of tumours of the central nervous system. Lyon, France: WHO/IARC; 2007. p. 33-53.

2. Gil-Salú J, Nieto A, Rodríguez JF, et al. Análisis de la presencia de mutaciones por pérdida de heterocigosidad de $1 p / 19 q$ en tumores cerebrales de estirpe glial. Neurocirugía. 2007;18:28593.

3. Zhou YH, Hess KR, Liu L, Linskey ME, Yung WK. Modeling prognosis for patients with malignant astrocytic gliomas: quantifying the expression of multiple genetic markers and clinical variables. Neuro Oncol. 2005;7(4):485-94.

4. Ohgaki H, Dessen $P$, Jourde $B$, Horstmann S, Nishikawa $T, D i$ Patre PL, et al. Genetic pathways to glioblastoma: a population based study. Can Res. 2004;64(19):6892-9.

5. Griffin CA, Burger $P$, Morsberger $L$, Yonescu R, Swierczynski $S$, Weingart JD, et al. Identification of $\operatorname{der}(1 ; 19)(q 10 ; p 10)$ in five oligodendrogliomas suggests mechanism of concurrent $1 p$ and $19 q$ loss. J Neuropathol Exp Neurol. 2006;65(10):988-94.

6. Jenkins RB, Blair $H$, Ballman KV, Giannini C, Arusell RM, Law $M$, et al. A t $(1 ; 19)(q 10 ; p 10)$ mediates the combined deletions of $1 p$ and $19 q$ and predicts a better prognosis of patients with oligodendroglioma. Cancer Res. 2006;66(20):9852-61.

7. Brat DJ, Seiferheld WF, Perry A, Hammond EH, Murray KJ, Schulsinger AR, et al. Analysis of 1p, 19q, 9p, and $10 q$ as prognostic markers for high-grade astrocytomas using fluorescence in situ hybridization on tissue microarrays from Radiation
Therapy Oncology Group trials. Neuro Oncol. 2004;6(2):96103.

8. Boulay JL, Miserez A, Zweifel C, Sivasankaran B, Kana V, Ghaffari $A$, et al. Loss of NOTCH2 positively predicts survival in subgroups of human glial brain tumors. PLoS One. 2007;2(6):e576.

9. Smith JS, Perry A, Borell TJ, Lee HK, O'Fallon J, Hosek SM, et al. Alterations of chromosome arms $1 p$ and $19 q$ as predictors of survival in oligodendrogliomas, astrocytomas, and mixed oligoastrocytomas. J Clin Oncol. 2000;18(3):636-45.

10. Cairncross JG, Ueki K, Zlatescu M, Lisle DK, Finkelstein DM, Hammond RR, et al. Specific genetic predictors of chemotherapeutic response and survival in patients with anaplastic oligodendrogliomas. J Natl Cancer Inst. 1998;90(19):1473-9.

11. Ichimura K, Vogazianou A, Liu L, Pearson DM, Bäcklund LM, Plant $K$, et al. 1 p36 is a preferential target of chromosome 1 deletions in astrocytic tumours and homozygously deleted in a subset of glioblastomas. Oncogene. 2008;27(14):2097-108.

12. Arslantas A, Artan $S$, Oner $U$, Müslümanoglu MH, Ozdemir $M$, Durmaz R, et al. Genomic alterations in low-grade, anaplastic astrocytomas and glioblastomas. Pathol Oncol Res. 2007;13(1):39-46.

13. Schmidt M, Antweiler S, Urban N, Mueller W, Kuklik A, Meyer-Puttlitz $B$, et al. Impact of genotype and morphology on the prognosis of glioblastoma. J Neuropathol Exp Neurol. 2002;61(4):321-8 
14. Pinto LW, Araújo MB, Wernersbach L, Leite AC, Chimelli LM, Vettore AL, et al. Glioblastomas: correlation between oligodendroglial components, genetic abnormalities, and prognosis. Virchows Arch. 2008;452(5):481-90.

15. Ishii $D$, Natsume $A$, Wakabayashi $T$, Hatano $H$, Asano $Y$, Takeuchi $\mathrm{H}$, et al. Efficacy of temozolomide is correlated with $1 p$ loss and methilation of the deoxyribonucleic acid repair gene MGMT in malignant gliomas. Neurol Med Chir. (Tokyo) 2007;47(8):341-9.

16. Krex D, Klink B, Hartmann $C$, von Deimling $A$, Pietsch $T$, Simon $M$, et al. Long-term survival with glioblastoma multiforme. Brain. 2007;130(Pt 10):2596-606.
17. Houillier C, Lejeune J, Benouaich A, Laigle-Donadey F, Criniere $E$, Mokhtari $\mathrm{K}$, et al. Prognostic impact of molecular markers in a series of 220 primary glioblastomas. Cancer. 2006;106(10):2218-23.

18. Gresner SM, Rieske P, Wozniak K, Piaskowski S, Jaskolski DJ, Skowronski W, et al. Gliomas: association of histology and molecular genetic analysis of chromosomes 1p, 10q, and 19q. Acta Neurobiol Exp (Wars). 2007;67(2):103-12.

19. Ligon $\mathrm{KL}$, Alberta JA, Kho AT, Weiss J, Kwaan $M R$, Nutt $C L$, et al. The oligodendroglial lineage marker OLIG2 is universally expressed in diffuse gliomas. J Neuropathol Exp Neurol. 2004;63(5):499-509. 